\title{
Spread of dye after single injection of transversus abdominis plane injection in adults. A cadaveric study
}

\author{
Ion Chesov, Elena Fatnic, Gheorghe Rojnoveanu, Adrian Belii
}

\section{Nicolae Testemitanu State University of Medicine and Pharmacy, Republic of Moldova}

\section{Background and Goal of the Study}

The transversus abdominis plane (TAP) block is a technique used for analgesia after abdominal wall surgery in adults. However, data on optimal injected dose regimen and volume are limited.

The goal of this study was to evaluate the character of dye spread in transversus abdominis plane, according to the volume of injected dye.

\section{Materials and Methods}

The Research Ethics Committee, Nicolae Testemitanu State University of Medicine and Pharmacy approved the study protocol.

The study was conducted on 15 fresh, unembalmed adult cadavers. Only subjects without acute abdominal diseases were enrolled, cause of death was other than abdominal pathology (cranio-cerebral, thoracic trauma, pulmonary embolism without abdominal pathology etc.).

Cadavers were divided into three groups (5 per group), according to the amount of dye injected $(10,20,40 \mathrm{~mL})$, Bilateral USG guided injection were performed for every singe cadaver. Injection point was in the mid axillary line in the space between costal margin and iliac crest.

After the dissection of the cadaver and the removal of the viscera, the spread of the dye was assessed on both side of each cadaver. The end points were the maximum length of dye spread in cephalo-caudal and medial-lateral direction.

For multiple comparison one way ANOVA was used, by pairs post-hoc analysis Duncan test was applied.

\section{Results}

All 15 cadavers were examined bilaterally (30 samples, 10 per group). There were no issues in USG location of transversus abdominis plane. The dye was present in all samples.

\section{Cefalo-caudal length of spread.}

Mean $(\mathrm{cm})$ for $40 \mathrm{~mL}$ was $-12.8(95 \% \mathrm{Cl} 11.7$ to 13.9$)$, without significant difference with $20 \mathrm{~mL}$ was $11.75(95 \% \mathrm{Cl} 10.5$ to 13.0$)$, $\mathrm{p}<0.109$, but significantly higher in comparison with $10 \mathrm{~mL}$ was 6.2 $(95 \% \mathrm{Cl} 5.5$ to 6.8$), \mathrm{p}=0,00006$. As well mean for $20 \mathrm{~cm}$ was significantly higher in comparison with $10 \mathrm{~mL}, p=0,00014$.

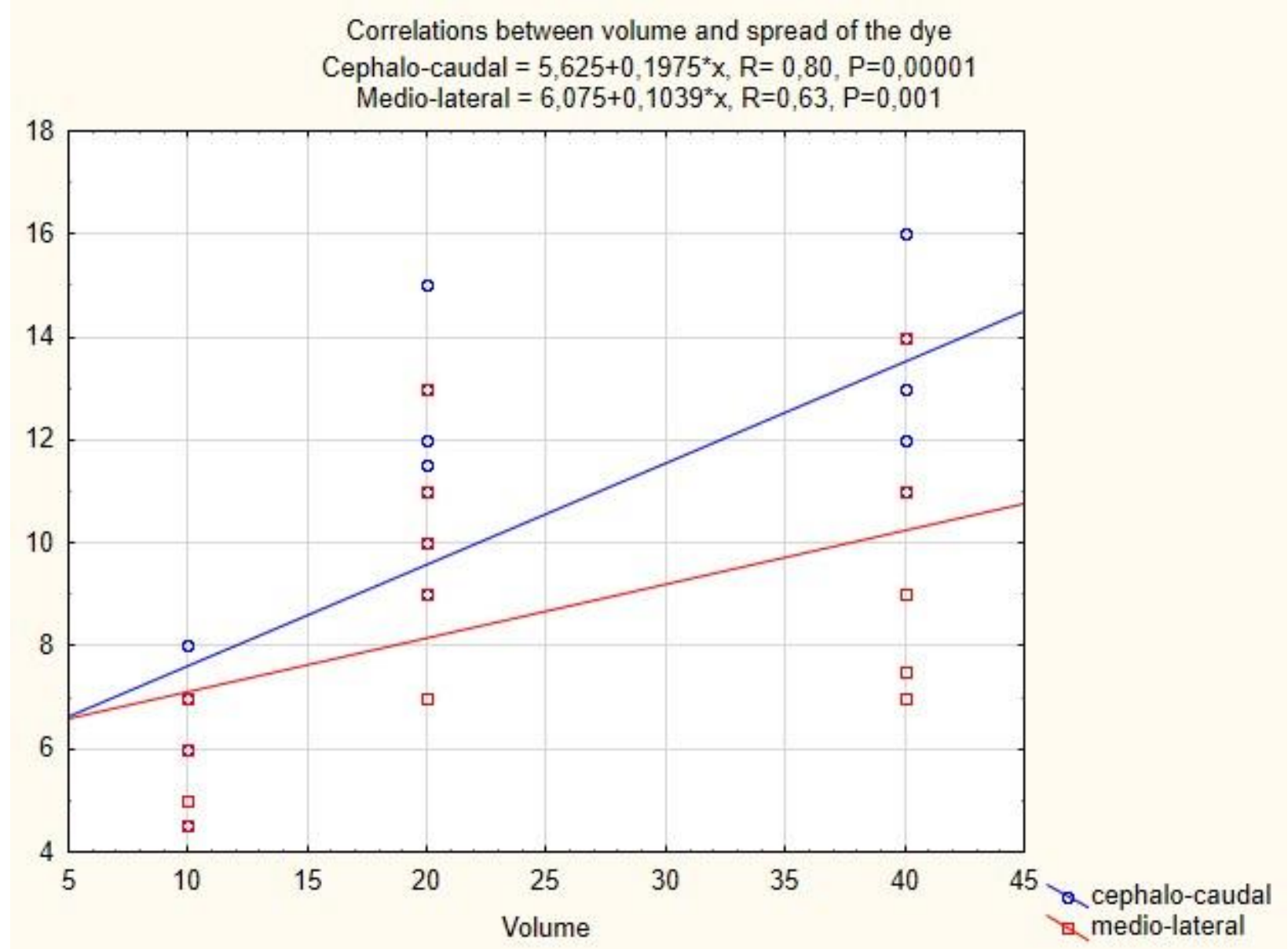

\section{Medio-lateral length of spread.}

Mean (cm) for $40 \mathrm{~mL}$ (Fig.1) was $9.6(95 \% \mathrm{Cl} 8.1$ to 10.9), without significant difference with $20 \mathrm{~mL}$ (Fig. 2) was $10.2(95 \%, \mathrm{Cl} 8.9$ to 11.5$), \mathrm{p}<0.109$, but significantly higher in comparison with $10 \mathrm{~mL}$ (Fig 3.) was $5.8(95 \% \mathrm{Cl} 5.1$ to 6.4$), \mathrm{p}=0.00015$. As well mean for $20 \mathrm{~cm}$ was significantly higher in comparison with $10 \mathrm{~mL}, \mathrm{p}=0,000065$.

\section{Conclusion}

The results of our studies show that there is no differences in the maximum length of cephalo-caudal and medio-lateral spread of the dye in the transversus abdominal plane after injection of a volume of 40 and $20 \mathrm{~mL}$.

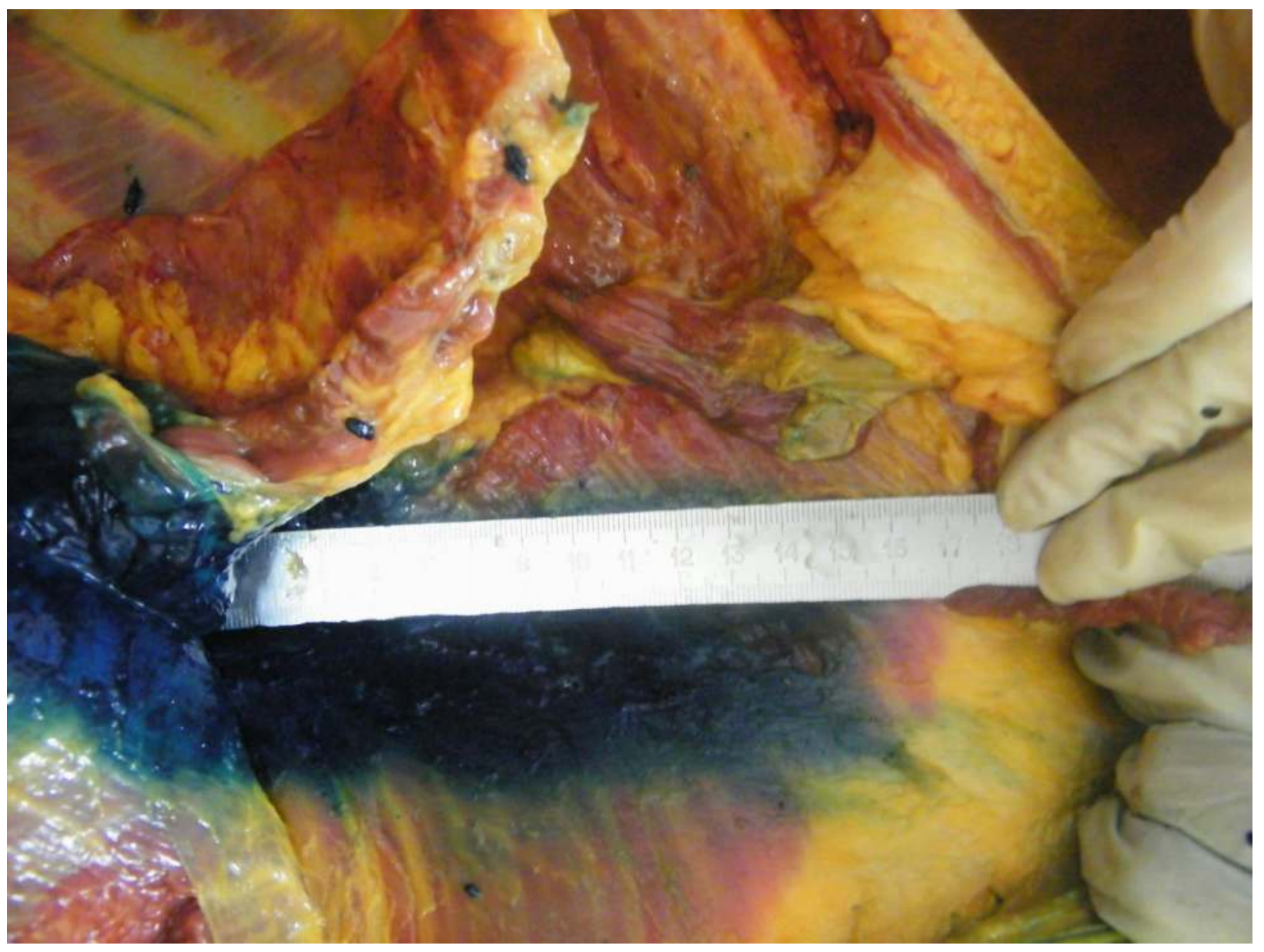

Fig. 1 Medio-lateral spread of the dye after $-40 \mathrm{~mL}$

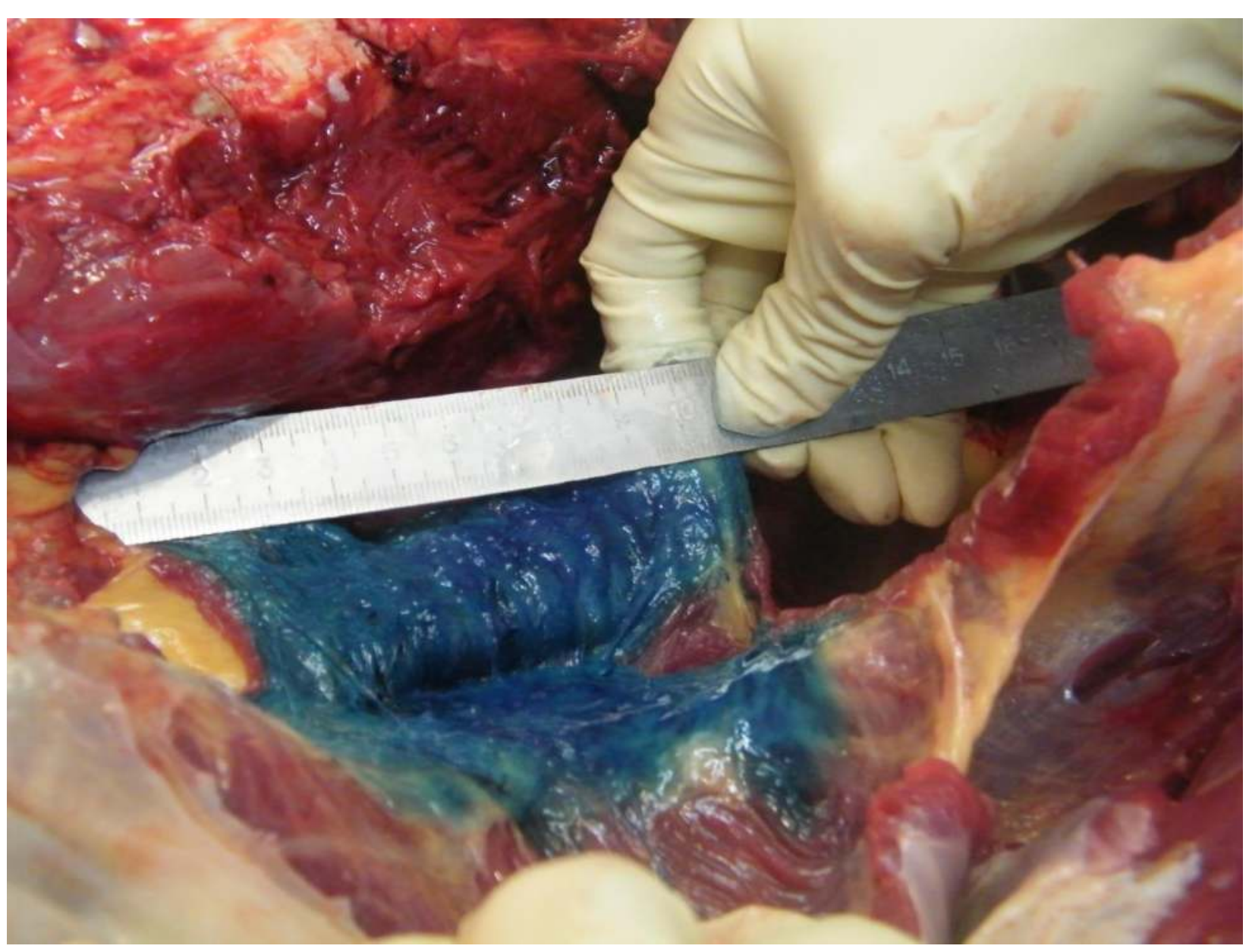

Fig. 2 Medio-lateral spread of the dye after $-20 \mathrm{~mL}$

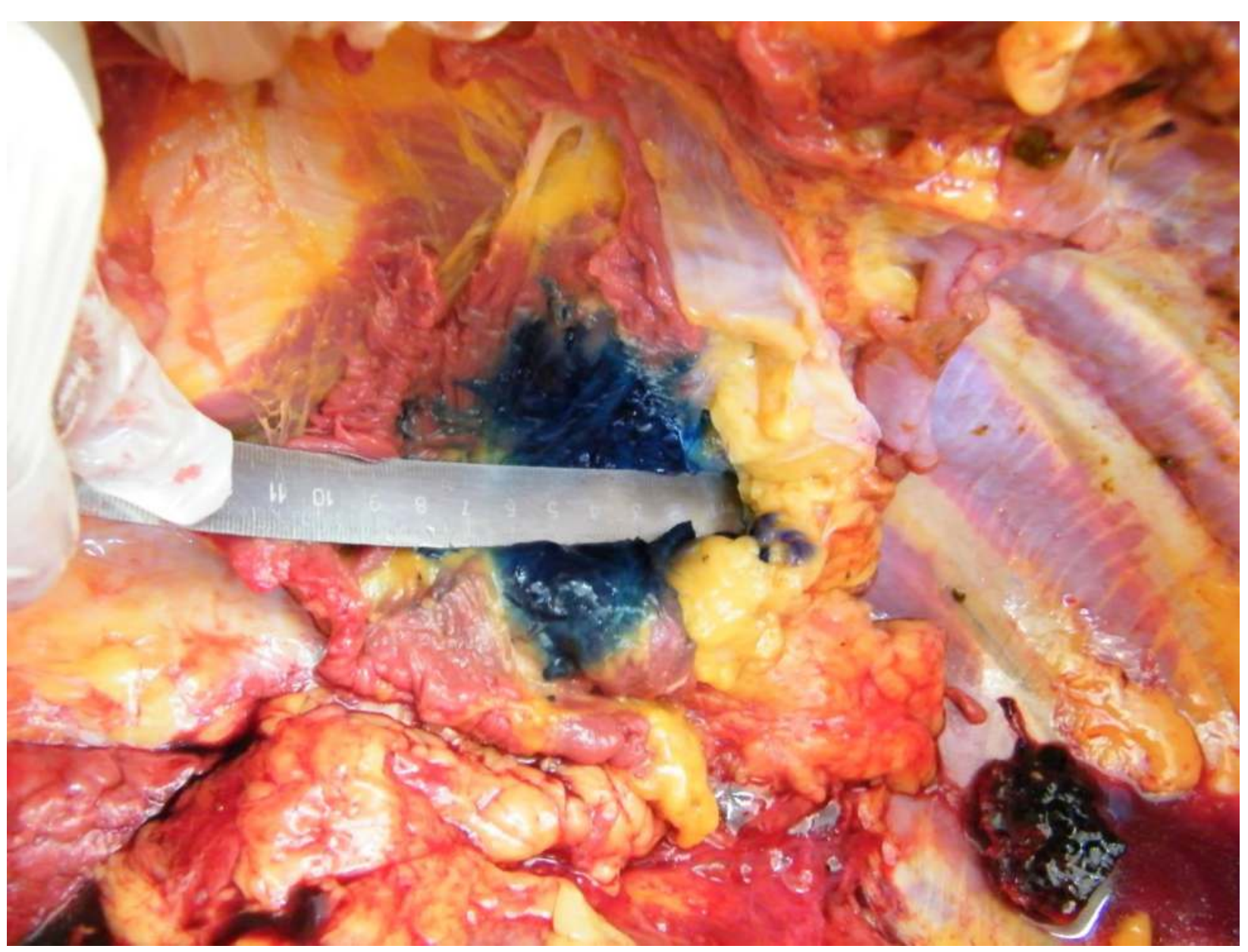

Fig. 3 Medio-lateral spread of the dye after $-10 \mathrm{~mL}$ 